\title{
Multiscale in modelling and validation for solar photovoltaics
}

Tareq Abu Hamed ${ }^{25}$, Nadja Adamovic ${ }^{1}$, Urs Aeberhard ${ }^{13}$, Diego Alonso-Alvarez ${ }^{50}$, Zoe Amin-Akhlaghi ${ }^{55}$, Matthias Auf der Maur ${ }^{26, *}$, Neil Beattie ${ }^{53}$, Nikola Bednar ${ }^{1}$, Kristian Berland ${ }^{31}$, Stefan Birner ${ }^{58}$, Marco Califano ${ }^{51}$, Ivana Capan ${ }^{4}$, Bostjan Cerne ${ }^{40}$, Irinela Chilibon ${ }^{35}$, James. P. Connolly ${ }^{10}$, Frederic Cortes Juan ${ }^{41}$, Jose Coutinho ${ }^{33}$ Christin David ${ }^{16}$, Knut Deppert ${ }^{44}$, Vesselin Donchev ${ }^{2}$, Marija Drev ${ }^{39}$, Boukje Ehlen ${ }^{29}$, Nicholas Ekins-Daukes ${ }^{50}$, Jacky Even ${ }^{11}$, Laurentiu Fara ${ }^{36}$, David Fuertes Marron ${ }^{42}$, Alessio Gagliardi ${ }^{15}$, Blas Garrido ${ }^{43}$, Violetta Gianneta ${ }^{17}$, Maria Gomes ${ }^{34}$, Jean-Francois Guillemoles ${ }^{12}$, Mircea Guina ${ }^{8}$, Janne Halme ${ }^{9}$, Mateja Hocevar ${ }^{38}$, Lucjan Jacak ${ }^{32}$, Witold Jacak ${ }^{32}$, Zoran Jaksic ${ }^{59}$, Lejo K. Joseph ${ }^{14}$, Spyridon Kassavetis ${ }^{20}$, Vaidotas Kazukauskas ${ }^{28}$, Jean-Paul Kleider ${ }^{10}$, Katarzyna Kluczyk ${ }^{32}$, Radovan Kopecek ${ }^{14}$, Ursa Opara Krasovec ${ }^{38}$, Jean-Louis Lazzari ${ }^{60}$, Efrat Lifshitz ${ }^{24}$, Martin Loncaric ${ }^{4}$, Søren Peder Madsen ${ }^{7}$, Antonio Marti Vega ${ }^{42}$, Denis Mencaraglia ${ }^{10}$, Maria E. Messing ${ }^{44}$, Felipe Murphy Armando ${ }^{23}$, Androula G. Nassiopoulou ${ }^{17}$, Ahmed Neijm ${ }^{52}$, Akos Nemcsics ${ }^{21}$, Victor Neto ${ }^{33}$, Laurent Pedesseau ${ }^{11}$, Clas Persson ${ }^{31}$, Konstantinos Petridis $^{18}$, Lacramioara Popescu ${ }^{14}$, Georg Pucker ${ }^{27}$, Jelena Radovanović ${ }^{37}$, Julio C. Rimada ${ }^{57}$, Mimoza Ristova ${ }^{54}$, Ivana Savic ${ }^{22}$, Hele Savin ${ }^{9}$, Marushka Sendova-Vassileva ${ }^{3}$, Abdurrahman Sengul ${ }^{48}$, José Silva ${ }^{34}$, Ullrich Steiner ${ }^{45}$, Jan Storch $^{5}$, Emmanuel Stratakis ${ }^{19}$, Shuxia Tao ${ }^{30}$, Pavel Tomanek ${ }^{6}$, Stanko Tomić ${ }^{49}$, Antti Tukiainen ${ }^{8}$, Rasit Turan $^{46}$, Jose Maria Ulloa ${ }^{42}$, Shengda Wang ${ }^{15}$, Fatma Yuksel ${ }^{47}$, Jaroslav Zadny ${ }^{5}$, and Javad Zarbakhsh ${ }^{56}$

1 TU Wien, Institute of Sensor and Actuator Systems,

Gusshausstr 27-29, 1040 Vienna, Austria

2 Faculty of Physics, Sofia University "St. Kliment Ohridski", Sofia, Bulgaria

${ }^{3}$ Central Laboratory of Solar Energy and New Energy Sources, Bulgarian Academy of Sciences, Sofia, Bulgaria

${ }^{4}$ Rudjer Boskovic Institute, Zagreb, Croatia

${ }^{5}$ Czech Academy of Sciences, Prague, Czech Republic

${ }^{6}$ Brno University of Technology, Brno, Czech Republic

7 Department of Engineering, Aarhus University, Aarhus, Denmark

8 Tampere University of Technology, Tampere, Finland

9 Aalto University, Espoo, Finland

${ }^{10}$ GeePs, UMR CNRS 8507, CentraleSupélec, Univ. Paris-Sud, Université Paris-Saclay, Sorbonne Universités, UPMC Univ Paris 06, France11 rue Joliot-Curie, 91192 Gif-sur-Yvette Cedex, France

${ }^{11}$ FOTON, INSA de Rennes, CNRS, UMR 6082, 35708 Rennes, France

12 CNRS, UMR IPVF 9006, Ile-de-France Photovoltaic Institute, 91130 Palaiseau, France and CNRS, NextPV, U. Tokyo, Japan

13 IEK-5 Photovoltaik, Forschungszentrum Juelich, 52425 Jülich, Germany

14 ISC Konstanz e.V., Rudolf-Diesel-Straße 15, 78467 Konstanz, Germany

15 Technische Universität München, Karlstrasse 45, 80333 Munich, Germany

${ }^{16}$ Madrid Institute for Advanced Studies in Nanoscience (IMDEA Nanoscience), C/Faraday 9, 28049 Madrid, Spain

17 NCSR Demokritos, Institute of Nanoscience and Nanotechnology, Athens, Greece

18 Technological Educational Institute of Crete, Chania, Greece

${ }^{19}$ Institute of Electronic Structure and Laser (IESL), Foundation for Research and Technology-Hellas (FORTH), 71110 Heraklion, Crete, Greece

20 Aristotle University of Thessaloniki, Thessaloniki, Greece

21 Obuda University, Budapest, Hungary

${ }^{22}$ Tyndall National Institute, Cork, Ireland

23 University College Cork, Cork, Ireland

24 Technion Schulich Faculty of Chemistry, Haifa, Israel

25 Dead Sea and Arava Science Center, Hevel Eilot, Israel

${ }^{26}$ Dept. Electronics Engineering, University of Rome Tor Vergata, 00133 Rome, Italy

${ }^{27}$ Fondazione Bruno Kessler, Trento, Italy

28 Vilnius University, Vilnius, Lithuania

\footnotetext{
* e-mail: auf.der.maur@ing.uniroma2.it
} which permits unrestricted use, distribution, and reproduction in any medium, provided the original work is properly cited. 
${ }^{29}$ Boukje.com Consulting, Bleiswijk, Netherlands

${ }^{30}$ Center for Computational Energy Research, Department of Applied Physics, Eindhoven University of Technology, Eindhoven, Netherlands

31 Department of Physics/Centre for Materials Science and Nanotechnology, University of Oslo, Oslo, Norway

${ }^{32}$ Wrocław University of Science and Technology, 50-370 Wrocław, Poland

${ }^{33}$ University of Aveiro, Aveiro, Portugal

34 Centre of Physics, University of Minho, Campus de Gualtar, 4710-057 Braga, Portugal

35 National Institute of Research and Development for Optoelectronics, INOE-2000, Department of Optospintronics, BucharestMagurele, Romania

36 University Politehnica of Bucharest, Bucharest, Romania

37 School of Electrical Engineering, University of Belgrade, Belgrade, Serbia

38 University of Ljubljana, Ljubljana, Slovenia

39 Trimo Group, Trebnje, Slovenia

40 ZEL-EN d.o.o., Trebnje, Slovenia

${ }^{41}$ Universitat Politecnica de Valencia,Valencia, Spain

${ }^{42}$ Universidad Politecnica de Madrid, Madrid, Spain

43 University of Barcelona, Barcelona, Spain

${ }^{44}$ Nano Lund, Lund University, Lund, Sweden

${ }^{45}$ Adolphe Merkle Institute, Fribourg, Switzerland

${ }^{46}$ Center for Solar Energy Research and Applications (GUNAM), Middle East Technical University, Ankara, Turkey

${ }^{47}$ Gebze Technical University, Kocaeli, Turkey

48 Bulent Ecevit University, Zonguldak, Turkey

49 Joule Physics Laboratory, School of Computing, Science \& Engineering, University of Salford, Manchester M5 4WT, UK

50 Imperial College London, London, UK

${ }^{51}$ University of Leeds, Leeds, UK

52 Silvaco Europe Ltd, St. Ives, UK

53 Northumbria University, Newcastle upon Tyne, NE1 8ST, UK

${ }^{54}$ Ss Cyril and Methodius University, Skopje, fYR Macedonia

55 ZAMSTEC - Science, Technology and Engineering Consulting, Arnoldstein, Austria

${ }^{56}$ Carinthia University of Applied Sciences, Europastrasse 4, 9524 Villach, Austria

57 PV Research Laboratory, Institute of Materials Science and Technology (IMRE) - Faculty of Physics, University of Havana, La Habana 10400, Cuba

58 nextnano GmbH, 85748 Garching bei München, Germany

${ }^{59}$ Centre of Microelectronic Technologies, Institute of Chemistry, Technology and Metallurgy, University of Belgrade, Belgrade, Serbia

60 Aix Marseille University CNRS, CINaM, Marseille, France

Received: 25 March 2018 / Received in final form: 13 July 2018 / Accepted: 18 July 2018

\begin{abstract}
Photovoltaics is amongst the most important technologies for renewable energy sources, and plays a key role in the development of a society with a smaller environmental footprint. Key parameters for solar cells are their energy conversion efficiency, their operating lifetime, and the cost of the energy obtained from a photovoltaic system compared to other sources. The optimization of these aspects involves the exploitation of new materials and development of novel solar cell concepts and designs. Both theoretical modeling and characterization of such devices require a comprehensive view including all scales from the atomic to the macroscopic and industrial scale. The different length scales of the electronic and optical degrees of freedoms specifically lead to an intrinsic need for multiscale simulation, which is accentuated in many advanced photovoltaics concepts including nanostructured regions. Therefore, multiscale modeling has found particular interest in the photovoltaics community, as a tool to advance the field beyond its current limits. In this article, we review the field of multiscale techniques applied to photovoltaics, and we discuss opportunities and remaining challenges.
\end{abstract}

Keywords: multi-scale modelling / solar cells / third generation photovoltaics / semiconductors / nano structures / device simulation

\section{Introduction}

The European Union (EU) and their public company sector are taking the leading role in the global challenge of increasing energy production from renewable sources. The $\mathrm{EU}$ is aiming to fulfill at least $20 \%$ of its total energy needs with renewables by 2020-to be achieved through the attainment of individual national targets [1,2]. All EU countries must also ensure that at least $10 \%$ of their transport fuels come from renewable sources by 2020. In its revised proposal for a "Directive Of The European Parliament And Of The Council On The Promotion Of 
The Use Of Energy From Renewable Sources" [3] the EU pledge to become a global leader in renewable energy and ensure that the target of at least $27 \%$ renewables in the final energy consumption in the EU by 2030 will be met. It was followed by the ambitious binding target, voted by the European Parliament in January 2018, that the renewable energy sources should account for $35 \%$ of total energy consumption by 2030 . The $10 \%$ bio-fuel target has also been revised to a $6 \%$ de-carbonization target in transport.

Since solar photovoltaics (PV) started to take on a globally significant role [4], the cost of PV power has fallen dramatically. Following reports of the International Energy Agency - Photovoltaic System Program (IEAPVPS) [5], the Renewable Energy Policy for the 21st Century (REN21) [6], and Joint Research Centre (JRC) of the European Commission (EC) [7], the PV market grew significantly in 2017. In total, at least $98 \mathrm{GW}$ of PV capacities were installed in the IEA PVPS countries and in other major markets during 2017. The total installed capacity in the IEA PVPS countries and key markets has risen to $402 \mathrm{GW}$. Solar PV technology continued to expand in 2017 thanks to the rapid development in China, India and some emerging markets. In the meantime, the US and Japanese market went down, while Europe experienced a slow rebirth partially hidden by the decline of the UK market. In other words, the global PV market outside of China grew by $4 \mathrm{GW}$ to $45 \mathrm{GW}$ while China drove the global numbers up to at least $96 \mathrm{GW}$. In the same way, the distributed PV market grew significantly for the first time since 2011 , with $38 \mathrm{GW}$ compared to $19 \mathrm{GW}$ one year before. More specifically, the cost of the solar cell (SC) by itself is no longer dominant in many terrestrial systems. For example, emerging organic photovoltaic (OPV) technology has the potential to provide cheap solar electricity, given advances in low-cost production and module efficiency and lifetime, and could compete with the established technologies in both roof- and ground-mounted systems if it can achieve a 10-year lifetime [8]. Renewable policies in many countries are moving from government-set tariffs to competitive auctions with long-term power purchase agreements. Increased competition has reduced remuneration levels for solar PV. This competitive price mechanism has squeezed costs along the entire value chain making tenders a cost-effective policy option for governments. Still, the average costs for solar PV remain relatively high. While auction need to be verified over time, they suggest that expanding competitive pricing could result in even lower average costs in coming years [9]. Somewhat counterintuitively, the rapid fall in costs is accelerating incentives in the EU to reach higher efficiencies as the quick growth of PV generated power globally increases its resources. Therefore, the race to close the gap between theoretical limits of solar cell efficiencies and those achieved at the laboratory scale and at industrial level has gained increased impetus.

In this context, research efforts in mainly three directions aim at increasing the cell efficiencies and reducing fabrication costs. Crystalline silicon (c-Si) is by far leading the PV market and there is still an intense research activity in Si based solar cells. Compared to the historical first generation c-Si solar cells that had a very simple design, with an n-type front emitter on a p-type c$\mathrm{Si}$ wafer along with an Al back surface field, significant improvements have been achieved introducing passivation schemes leading to the so-called PERC (Passivated Emitter and Rear Cell) and PERL (Passivated Emitter, Rear Locally-diffused) cells with a record efficiency of $24.7 \%$ (that has been reevaluated to $25 \%$ ) [10] obtained on a small size cell $\left(\right.$ few $\left.\mathrm{cm}^{2}\right)$. This record has been unbeaten for more than 15 years, but it has been broken several times recently using various new concepts, namely the interdigitated back contact cell (IBC) architecture [11], and, very recently, the so-called TopCon concept, tunnel-oxide passivated rear contact and high-quality top surface passivation [12]. Another very powerful concept is the silicon heterojunction (SHJ) combining crystalline silicon with very thin layers of hydrogenated amorphous silicon (a-Si:H). Doped a-Si:H, p-type and n-type, is used to produce the front emitter junction and the back surface field, respectively, on an n-type crystalline silicon absorber. A very thin undoped a-Si:H (so-called intrinsic) is inserted between the doped a-Si:H layers and the c-Si wafer to achieve outstanding surface passivation. Double side contacted SHJ solar cells have demonstrated efficiencies of $25.1 \%$ on $160 \mu \mathrm{m}$ thick c-Si [13], and record open circuit voltages of $750 \mathrm{mV}$ on $100 \mu \mathrm{m}$ thick cSi [14]. Finally, the present record efficiencies are held by a technology combining the interdigitated back contact structure with the SHJ concept. A value of $26.3 \%$ has been published in [15], while a record value of $26.6 \%$ has been recorded in the best research-cell efficiency chart from the US National Renewable Energy Laboratory (NREL) [16].

The second research direction, designated as the 2nd solar cell generation, pursues technologies that hold promise of major advances in costs and fabrication. These include notably thin films and organic PVs, for large-area fabrication with low material and fabrication costs.

The last direction, known as 3rd solar cell generation, is aiming at technologies promising major advances in efficiencies to overcome the Shockley-Queisser limit [17]. In fact, conventional single energy gap SCs have an ultimate efficiency limit that was established by Shockley and Queisser based on detailed balance arguments. The "balance" in the model comes from the fact that it quantitatively accounts for two opposing fundamental processes that occur in any SC: absorption and emission. For a $\mathrm{SC}$ at room temperature the maximum efficiency is further reduced to $40.7 \%$ for a band gap of $E_{\mathrm{g}}=1.1 \mathrm{eV}$ under maximum concentration condition, and to $31 \%$ with $E_{\mathrm{g}}=1.3 \mathrm{eV}$, at one sun (i.e., when the solid angle subtended by the sun shining on a cell at normal incidence is taken into account). The main reason underlying those values is that only photons with an energy close to that of the semiconductor band gap are effectively converted. Photons with lower energy than $E_{\mathrm{g}}$ are simply lost (the semiconductor is transparent to them), and photons with higher energy $\left(>E_{\mathrm{g}}\right)$ convert their energy at best partially into electricity, wasting the excess energy into heat.

A number of concepts that all share the goal of managing thermalization and optical losses have been devised. These range from multi-junction solar cells 


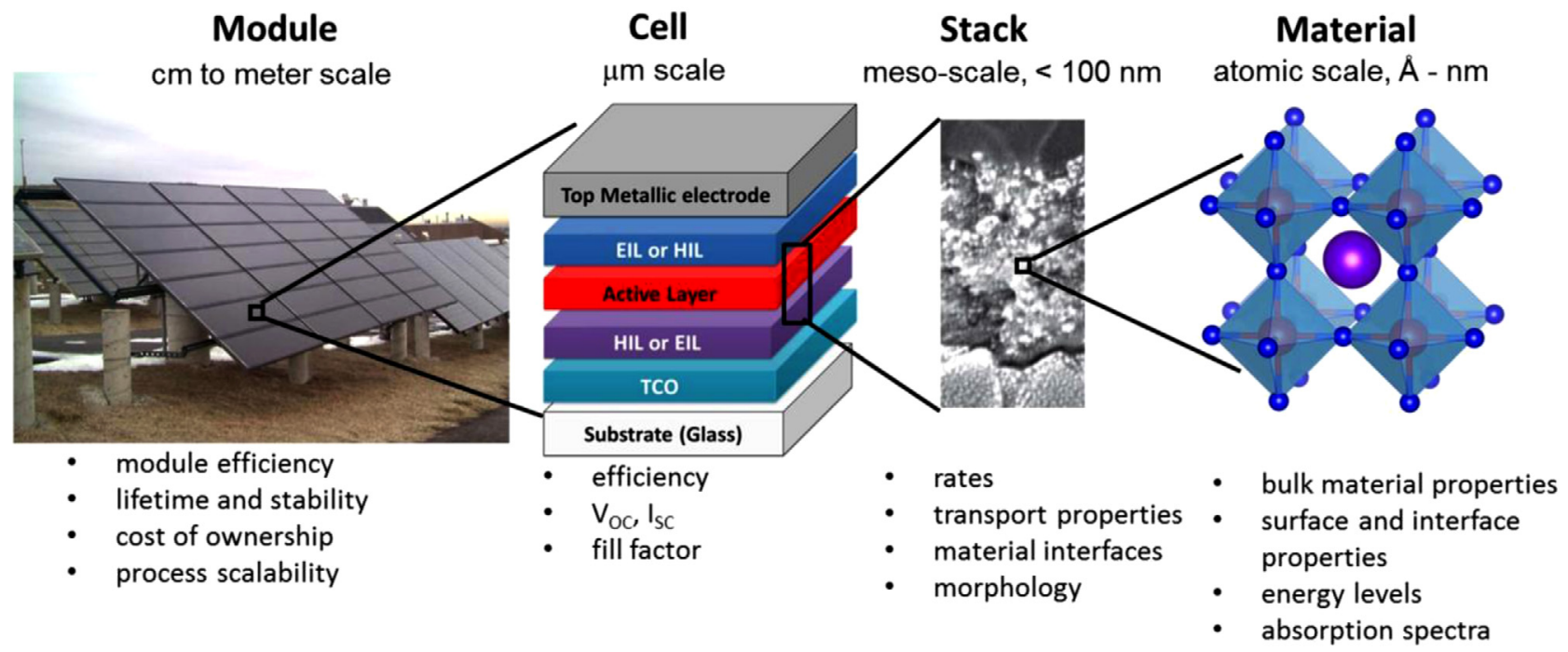

Fig. 1. Scales of interest in photovoltaics technology and respective quantities of interest.

(MJSC), which have proved record efficiencies higher than the Shockley-Queisser limit [18], to the research exploiting novel materials and concepts such as the class of nanostructured cells in the fields of hot carrier cells, intermediate band solar cells (IBSC), multiexciton-generation solar cells (MEG), or luminescent solar concentrators (LSC), to name a few, which conceptually promise enormous potential. Their development into real devices is spurring the competition between leading experimental groups worldwide.

All these research directions are heavily guided and supported by tremendous advances in theory and computer modeling. There has been much progress in all relevant computational modeling approaches, ranging from ab initio evaluation of material properties [19], to mesoscale methods describing nanoscale structures and their dynamical electronic and optical properties including processes at interfaces [20,21], to combining these concepts at the device scale [22,23], and finally industrial applications thereof. More generally, the importance of nanostructures in scientific and industrial applications has been constantly increasing during the last decades. The study of the specific behavior of such structures in terms of electronic, optical and thermal properties and its influence on macroscopic device characteristics requires the combination of modeling and characterization techniques at different scales. Indeed, there has been much effort in sharing lessons learned across this range of physical scales, and the resulting field of multiscale modeling and characterization is very active [23-25]. Figure 1 shows schematically the main four scales involved, which reach from the atomistic scale up to module level.

Despite major efforts in the development of novel PV concepts, the efficiency reached in their practical implementation is most often substantially lower than what is estimated by theory. Thus, an increased level of realism especially concerning unavoidable losses associated with nanostructures is one of the motivations driving the multiscale simulation efforts in this field.
In response to this, the MultiscaleSolar COST Action network was established in 2015 [25,26] bringing together academic and industrial partners. It is structured into four workgroups reflecting atomistic, mesoscopic, device and industrial scales. The main aim of the network is to explore the challenging implementation of next generation solar cell architectures that require novel multiscale modeling and characterization approaches that capture both the peculiar features at the nanoscale and their impact on the optoelectronic performance at device level.

In this review, we discuss ongoing trends and remaining challenges on the different length scales including active research directions in the network. Therefore, the paper is organized as follows: We first introduce the four distinct scales sketched above and their respective main issues and difficulties to be addressed by the research community, and then give details of selected topics that have evolved into particularly intense activity in MultiscaleSolar.

\section{From atomic to industrial scale}

\subsection{Atomic scale and nanostructure states}

The understanding of materials for photovoltaics at the atomic level, and parameterization strategies at the mesoscopic scale to further study mesoscopic carrier dynamics in nanostructures on the next length scale is the first crucial step for a multiscalar approach. Modeling on prospective materials or designs is also targeted to improve basic material properties in order to find new strategies for cost reduction leading to next generation solar cells. The main semiconductor materials for PV are studied, including silicon, III-V semiconductors, chalcopyrites, and the halide perovskites.

Different atomistic approaches are used to clarify bulk material properties over different phases, and at surfaces and material interfaces [27]. One has to choose a specific 
level of theory depending on the targeted property. In most cases, the density functional theory (DFT) [28] is a tool well-suited to characterize the mechanical or vibrational properties of the bulk material. Concerning electronic properties, a more sophisticated level of theory is added on top of the DFT such as hybrid corrections, either addressing the nonlocality of fermionic interaction (B3LYP) $[29,30]$, or screening the long-range Coulomb interaction (HSE) [31] or even better many body corrections (GW) [32]. Finally, the optical properties are strictly obtained by using the time dependent DFT (TDDFT) [33] or DFT plus HSE/GW and on top the Bethe-Salpeter corrections (BSE) [34], with recent results on new photovoltaic materials [35]. In parallel, the simplified, so-called DFT-1/2 method, an approximate quasiparticle method [36], is explored. This methodology is computationally less demanding compared to the HSE or GW many-body corrections. Very recent progress [36] shows that DFT- $1 / 2$ method yields accurate band gaps of hybrid perovskites with the precision of the GW method with no more computational cost than standard DFT. This opens the possibilities of accurate electronic structure prediction of sophisticated halide perovskite structures and new materials design for lead free materials. In practice, various and massively parallel codes e.g. VASP [37,38], ABINIT [39], SIESTA [40], Quantum-Expresso [41], CRYSTAL [42] and CASTEP [43] are used to carry out such simulations.

The strategy for the discovery of new materials, such as the $2 \mathrm{D}, 2 \mathrm{D} / 3 \mathrm{D}$, and $3 \mathrm{D}$ hybrid perovskite halides, is based on an accurate description of the electronic properties (band structures) for academic cases such as $2 \mathrm{H}-\mathrm{PbI}_{2}$, $\mathrm{CsPbI}_{3}$ and $\mathrm{MAPbI}_{3}$. Based on our previous studies [4447], the most accurate level of theory (DFT $+\mathrm{SOC}+\mathrm{HSE}$ or $\mathrm{DFT}+\mathrm{SOC}+\mathrm{GW}$ if possible) will be necessary. In parallel, a comparison between the benchmark results on these materials is mandatory to validate the DFT- $1 / 2$ method for this class of materials. A preliminary study is underway. However, close to the band gap energy, it already starts to reveal a good accordance on the band structures and more on the effective masses. If the comparison is a success, a huge execution time improvement will be achieved.

For the industrially most relevant case of the SHJ solar cell, the amorphous component of the interface region has to be created using ab initio molecular dynamics (MD) prior to characterization by DFT methods, and hightemperature annealing via MD drastically affects the coordination of the atoms and resulting density of localized gap states at the interface [48].

Other subjects explored relate to absorbing materials for thin film solar cells and transparent conducting oxides (TCO) $[49,50]$. The strategy for doping, formation of impurities, stable and ordered defective phases, dielectric, and optical properties of technologically important $\mathrm{Cu}$ chalcogenides, either in chalcopyrite or wurtzite phase, is examined at the level of theory that encompasses DFT + $\mathrm{HF}+\mathrm{BYLYP}$ or $\mathrm{DFT}+\mathrm{sXC}+\mathrm{SCO}+\mathrm{TDDFT}$ [51-53], or even GW [54]. A similar methodology is used for reduced graphene oxide and similar $2 \mathrm{D}$ allotropes as materials for TCO [55].
In our overall strategy, for the wide range of absorbing materials relevant for PV applications, a campaign to parameterize semi-empirical (k $\cdot \mathbf{p}$ or tight-binding) [56-58] simulation has been proposed. The DFT plus GW corrections helps to get the correct offset of the heterostructure through the Van de Walle and Martin method [59]. Moreover, the energy gaps, optical dipoles, deformation potentials, elastic and piezoelectric properties will be extracted by DFT [60]. In this task the kppw code, parallel implementation and symmetry adapted [61], plane waves based $\mathbf{k} \cdot \mathbf{p}$ code [53] and the real space parallel $\mathbf{k} \cdot \mathbf{p}$ code as implemented in TiberCAD [62] will be used. Our efforts to parameterize $\mathbf{k} \cdot \mathbf{p}$ Hamiltonian has also lead to a new Brillouin zone interpolation scheme, which is being used to resolve fine features of the dielectric functions with drastically reduced computational costs [63]. Another objective is to calculate carrier mobilities including electron-phonon interactions, e.g. in $\mathrm{PbTe}$, and other materials [64].

Complementing this, expertise in tight-binding modeling $[65,62,66-68]$ is used to build empirical Hamiltonians for novel materials and heterostructures. Input from DFT calculations such as piezoelectric tensors can also be introduced to simulate the electromechanical properties of these heterostructures. Simple hybrid perovskite halide materials serve as an academic study on this point.

\subsection{Mesoscopic carrier dynamics in nanostructures}

On the mesoscale, carrier dynamics in nanostructures addresses the critical impact of nanostructure-based solar cell device components on the performance of the entire photovoltaic device. Since on the one hand, nanostructure properties depend on the actual microscopic configuration in terms of size, shape and composition, and on the other hand, they need to be propagated to the device level in order to assess the impact on device characteristics, multiscale approaches are crucial in both implementation and exploitation of mesoscale simulation and characterization.

The role of the mesoscale dynamics as the linking element between microscale material properties and the macroscale device characteristics defines natural interfaces between research groups working on the atomic and device scales, respectively. On the side of the atomistic activities, local mesoscopic models are parameterized from microscopic information in terms of basis functions for the representation of mesoscopic Hamiltonians. The mesoscopic models form the basis to compute local rates for different dynamical processes involving nanostructure states, such as generation, recombination and transport of charge carriers, relying on (quantum)-kinetic methods such as the non-equilibrium Green's function formalism (NEGF) [69] or kinetic Monte Carlo (KMC) [70], but also basic application of Fermi's golden rule. On the macroscale side, reviewed in the next section, the local rates and mobilities are inserted in macroscopic continuum models for electronic transport such as the standard driftdiffusion-Poisson equations coupled to Maxwell solvers for the light propagation, which allow for the multi-physics modeling of realistically extended solar cell device 
structures including complex contact geometries or absorber morphologies. In terms of experimental characterization, the mesoscale information concerns the nanostructure aspects (in contrast to atomically resolved or bulk properties), and can be used for both validation and parameterization of (empirical) mesoscale models in the case where ab initio parameterization is not available.

Several different third-generation or high-efficiency photovoltaic device concepts with implementation approaches based on nanostructure components are currently investigated. For example, there are ongoing activities on mesoscale carrier dynamics in the context of the SHJ solar cell, III-V quantum dot solar cells (QDSC) for multi-junction and intermediate band (IB) applications and in organic photovoltaics (OPV).

In the SHJ case, the nanoscale region of the interface between amorphous and crystalline silicon with decisive impact on the device properties is created atomistically and from first principles using ab initio molecular dynamics, and the electronic structure is evaluated with density functional theory [48]. The charge transport and recombination across the interface is simulated using NEGF, and complex interdigitated contact configuration as well as light management via textures and antireflection coatings are considered in an integrated 3D TCAD approach [24].

For QDSC, the electronic structure of InAs-GaAs quantum dots is determined using microscopic continuum $\mathbf{k} \cdot \mathbf{p}$, plane wave based methodology [71], which provides a coarse-grained localized basis in terms of quantum dot Wannier functions [72]. The mesoscopic Hamiltonian of the finite quantum dot array using such a basis captures both the effects of dot-to-dot variation in the couplings and the impact of contacts [73], and it provides both carrier dynamics and device characteristics at the mesoscale via the NEGF formalism.

In the OPV application, the complex organic blend morphologies can be generated via the Metropolis Monte Carlo technique [74,75]. Subsequently, effective hopping rates, carrier lifetimes and mobilities can be extracted from KMC simulations and inserted into a macroscopic driftdiffusion-Poisson solver, thus linking from mesoscopic to macroscopic scales [76]. The electronic structure and polarization at organic interfaces can also be studied on ab initio level using the charge patching method within DFT [77] or DFT-based tight-binding [78], and exciton formation as well as ultra-fast separation of photogenerated charge carriers at such interfaces have been assessed based on a density matrix formalism [79,80].

On the characterization side, surface photovoltage spectroscopy is applied to the study of the absorption of dilute nitride films with application in multijunction devices and compared to both photoluminescence (PL) and the dielectric response as provided by Fermi‘s Golden Rule based on electronic structure computation in the empirical tight-binding approach [81,82]. In addition, dilute nitrides have been studied by X-ray photoelectron spectroscopy (XPS), Raman and PL spectroscopy to determine the degree of atomic ordering in the quaternary alloy InGaAsN during the liquid phase epitaxial growth at near thermodynamic equilibrium conditions and its influence on the band gap formation [83].

\subsection{Macroscopic device characteristics}

The third length scale studies solar cell characteristics at the macroscopic that is on device level both from a theoretical and characterization point of view. Modeling of PV devices is performed using different types of models, ranging from physics based Technology CAD simulations to analytic models. Special focus is given to the definition of multiscale strategies, in particular coupling device scale modeling to the modeling of $\mathrm{PV}$ materials performed at mesoscopic scale, in order to develop simulation approaches that can provide a higher degree of accuracy and predictability. This is particularly important in 3rd generation $\mathrm{PV}$, which includes a range of concepts. For example, possible polarization domains or mobile lattice defects in hybrid perovskites need to be taken into account, but also particular properties of nanostructures like quantum dots, such as carrier confinement and modified scattering rates, need to be considered in device models. Similarly, complex morphologies for enhancing light absorption and carrier generation (such as in organic bulk-heterojuntion solar cells), and novel concepts based on intermediate bands, hot carrier generation or multiexciton generation, which are not modeled out-of-the-box in current commercially available device simulation software, require special care and a combination of modeling on multiple scales.

Several TCAD software industrial providers with interest in multiscale and multiphysics approaches, among which are Silvaco [84], Nextnano [85] and TiberLab [86], are actively involved in solar cell device simulations and in the development of linking approaches to the mesoscale dynamics as described in the previous section. This is not limited to electronic transport alone, but the concept is also applied to optical device performance and light management.

A series of specific issues on the macroscopic device characteristics level have been identified in the PV community. Currently under study are, for example, modeling and characterization of $\mathrm{c}-\mathrm{Si}$ based $\mathrm{PV}$ devices and tandem including device architectures combining c- $\mathrm{Si}$ and Si nanowire solar cells [87], tandems with metal oxide cells on top of Si cells [88], and potentially other tandem architectures. A cross-cutting major issues is the characterization and modeling of defects, which is also a multiscale axis of interaction with research on other length scales $[89,90]$.

Of particular interest in the field of organic photovoltaics is the coupling to the mesoscopic and microscopic modeling in order to obtain reliable material parameters such as densities of states, electronic and excitonic transport parameters and interface related properties. For bulk heterojunctions specifically, there is a need to include the effects of the morphology of the material blend in device level simulations beyond the effective medium approximation [91]. Further, extraction of transport and carrier dynamics parameters from kinetic Monte Carlo models (KMC), or concurrent coupling of KMC with semi-classical drift-diffusion type models to study local details in full, potentially $3 \mathrm{D}$ device structures is of interest. 
The study and exploitation of optical effects such as plasmonic enhancement, scattering at nanostructured particle layers and up-/down-conversion for PV applications both from an experimental and theoretical perspective has raised much interest. Modeling is in particular related to improve the description of quantum effects in plasmonic structures using the input from atomistic models. Objects of study are for example regularly or randomly ordered dispersed nanoparticle distributions, or nanowires for light management.

Furthermore, activity is underway on modeling of quantum confined structures by combining mesoscopic, quantum kinetic models with semi-classical or analytical models. This includes for example the combination of nonequilibrium Green's function based approaches with driftdiffusion models, for a locally accurate description of carriers in quantum confined regions.

One of our goals when dealing with macroscopic device characteristics is to define the interfaces to link to the mesoscopic and atomistic models. This will allow the implementation of device level modeling approaches including microscopic details, necessary to fully assess the potential of 3rd generation PV concepts, in a similar way as has been demonstrated e.g. for III-nitride based LEDs [92] or quantum information processing (QIP) devices [93]. It will then be possible to calculate the key solar cell performance parameters needed at module level to evaluate the industrial perspectives discussed next of the different PV concepts.

\subsection{Industrial perspectives}

The status and evolution of different PV technologies, their corresponding markets and implementation plans are addressed through a number of reports, roadmaps, and white books. The most relevant are those published by the European PV Industry Association (now SolarPower Europe), the European PV technology Platform (ETIP$\mathrm{PV}$ ), national implementation plans (such as the ADEME Roadmap in France), the Global PV Industry \& Technology Platform (SolarUnited, former International Photovoltaic Equipment Association IPVEA), and PV program of the International Energy Agency (IEA PVPS).

For instance, the 2017 SET-Plan Declaration on strategic targets of ETIP-PV in the context of an initiative for global leadership in photovoltaics includes an implementation plan that contains concrete R\&I activities for achieving these targets. The scope of these R\&I activities is however large and covers the whole value chain from advanced materials and technologies to multiscale system integration and usages. All roadmaps point to the need to pursue intense research on silicon technologies (PERC, HIT, PERL, IBC) to increase the efficiency up to the Shockley-Queisser limit for a single junction. Even if the cost of production of the photovoltaic modules continues to drop, and even if the cost of installation makes PV a competitive energy, each percentage of yield and each year of lifetime earned remains a criterion for photovoltaic operation. From this point of view, modeling makes it possible to realistically determine the functional characteristics of silicon solar cells and modules, but is not able to predict their evolution over time. For quality and reliability issues, it is then necessary to use standardized tests and experimental test-error methods.

In order to be predictive enough and therefore useful for industrial end-users, simulation and modeling efforts are critically depending on input from industrial partners, both for established and emerging PV technologies. It is therefore important to build bridges between academia and industry for mutual exchanges on which technologies hold industrial promise, what the latest technical and industrial developments are, and which partnerships to build. This delivers vital feedback to the research community. One of the aims of this industry oriented research collaboration is to gather and exchange technical data, best practices, and standards in order to yield multiscale modeling of the structures recommended for study. It allows also a coordination of multiscale characterization in order to validate the modeling methods and approaches developed.

MultiscaleSolar has applied COST short term missions to these ends by allowing researchers to investigate academic and industrial priorities on site in academia and in industry. Research has involved formal exchanges, interviews, online questionnaires/survey and other methods known in social science and market research studies. These outreach research activities connect the photovoltaic community, industries and their customers to understand their products, services, requirements and visions. Thus, PV roadmaps and the management of costs in fabrication can be improved with the help of a large number of experts. While gathering high impact knowledge in closely related fields, we also spread these acquired skills and discoveries to increase general awareness. A key means to achieving this impact is through our industrial collaborators which span multinationals such as Electricité de France (EDF) [94,95], Toyota [96], Silvaco [84] and Horiba [97], to small and medium size enterprises such as Solar Capture Technologies (UK) [98], nextnano GmbH (Germany) [85], TiberLab Srl (Italy) [86], 3GSolar Photovoltaics Ltd. [99], BISol Production Ltd. [100], SemiLab Ltd. [101] and start-ups including Quantum Base Ltd. (UK) [102], NTS NanoTechnologySolar GmbH (Germany) [103].

Industry-near projects such as light optimization, 3D printed optics, characterization on module level and modeling activities, such as optical (Ray-Tracing) and photonic (FEM, FDTD, PWE) simulations can be coordinated by partners at the interface between fundamental research and industry to close the research and marketing gaps. This includes keeping track of the impact of recent research results on simulation, design, fabrication and applications, and also scrutinizing promising 3rd generation concepts with respect to their industrial feasibility and possible commercialization.

From these activities primarily progress in optical aspects has resulted. This involves fabrication methods on the one hand, with research on 3D printing developed by academic MultiscaleSolar partners in particular being singled out as a strong candidate for implementation in industrial contexts. On the optical front, the outreach 
research missions in industry have been complemented by technical research missions. One example we want to single out is a mission on the links between structural and optical disorder in distributed Bragg reflectors. This work has yielded methods to characterize imperfections in optical resonators of great interest for industry in $\mathrm{PV}$ and beyond.

A second example related to building integrated PV (BIPV) is the development of optimal color-forming structures for maximal efficiency colored photovoltaics. This showcases the exchange between the mesoscale optical modelling activities, the device level consequences, and the application to industry, involving on-site development of the concept with industrial partners. This work has been submitted to the IEEE Journal of Light Wave Technology.

A concluding recent example of excellence in applied research emerging from our Action is significant progress in using inkjet printing to accelerate the process of attaching light absorbing dyes to a nanocrystalline $\mathrm{TiO}_{2}$ photoelectrode [104].

\subsection{Selected activities in MultiscaleSolar}

In the following sections, we present several specific research directions within the network. Activities across all material platforms have emerged. For organic solar cells, we evaluate solar cell performance using a nanosized, intermixed morphology of acceptor and donor materials simulated with Monte Carlo and finite element methods. We discuss research on intermediate band gap cells and give a summary on ongoing optimization and design studies of multijunction solar cells. A further emphasis is put on the study of CdSe/CdTe type-II QDs based solar cells for multiple exciton generation. Optical properties of solar cells employing nanostructured front surface layers made from dielectric and plasmonic materials are studied with Rigorous Coupled Wave Analysis enhanced with results from microscopic theories regarding strong coupling and other non-classical effects.

\subsection{Simulation of organic solar cells including bulk heterojunction morphology}

Today's organic solar cells (OSC) are characterized by a complex interface between donor and acceptor materials in order to efficiently split the photogenerated excitons in the organic semiconductors [105]. This is known as the bulkheterojunction architecture (BHJ). Similarly, in dye sensitized solar cells (DSCs) two materials, an electron and hole transporter, respectively, are intermixed and at the common interface a molecular dye is inserted in order to absorb light. Finally, perovskite solar cells often have a mesoporous layer, which seems to improve stability [106]. All three device types share a common feature: the active layer is partially or totally composed by intermixing two materials, which have a mutual complex interface where fundamental processes take place. In an OSC, for example, the interface is where photogenerated excitons split into free charge carriers, but also where undesired recombination processes occur.
A fundamental challenge for device efficiency in BHJ is to find and control the optimal phase separation scale for exciton dissociation interfaces and charge transport channels. In many cases microscopic investigations on a working device is experimentally challenging. As a result, simulation comes into play for verifying the proposed models and guiding the device optimization. One way to model charge transport properties in such devices is to solve the drift-diffusion (DD) equations [91,107]. They offer an approach at a macroscopic continuum level, low computational effort, and with good agreement to experimental data.

The multiscale aspect in this specific type of simulation is given by a suitable inclusion of the real blend morphology in the macroscopic device simulation, which goes beyond the commonly used effective medium approximation (EMA). The latter treats different intermixed materials in the same region as one effective material $[108,109]$. However, as shown in [110], EMA may introduce drastic approximations. The reason is that the internal interface plays a fundamental role in BHJ solar cells and effective medium approximations completely neglect this fact. Therefore, a tool has been implemented in the MultiscaleSolar network to generate a model of the real internal morphology of a BHJ which can be combined with a DD model to simulate the device including the real internal structure of the blend $[110,111]$.

One of the key issues is the generation of a suitable morphology and its subdivision into polyhedra for subsequent finite element analysis. To generate the morphology of an arbitrary, randomly intermixing blend a simple stochastic method commonly used for kinetic Monte Carlo simulation has been adopted [70,112]. A 3-dimensional spin system, where equal number of spin-up and spin-down represent the two materials, respectively, is annealed using a Metropolis Monte Carlo algorithm.

In such an approach, the average size of nucleated spin clusters can be tuned by limiting the number of spin-swap steps. The resulting morphology (referred to as MMC morphology hereafter) needs to be repaired and optimized for the sake of numerical simulation. This is achieved sequentially on voxel level and mesh level. On voxel level, the raw MMC morphology is cleared via the removal of island spins and condensing spurious spins at the cluster boundaries. Furthermore, features like sharp corners or thin wedges near the volume boundary are eliminated by suitable algorithms.

Next, the interface (iso-surface) of the MMC morphology is extracted using a Marching Cubes algorithm, resulting in a triangle mesh. Optimization at the mesh level is performed by smearing out the bumpiness using a modified Laplacian smoothing algorithm, which preserves the volumes and prevents from mesh distortions [113,114]. After smoothing, the interface is further remeshed mainly for two purposes: 1. resampling the mesh for controlling the mesh resolution, and 2 . improving the mesh quality, i.e. making triangles as equilateral as possible. To generate finally the 3-dimensional finite elements on the modeled geometry, the mesh morphology can be readily fed into the majority of finite element mesh generators, such as Gmsh [115]. 
a)

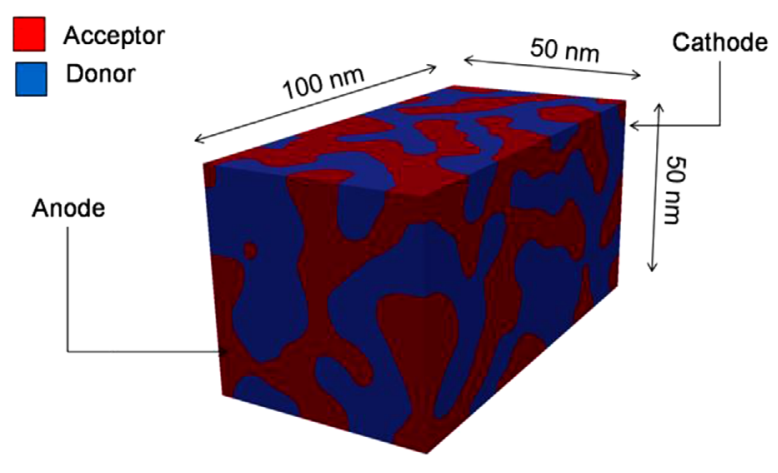

b)

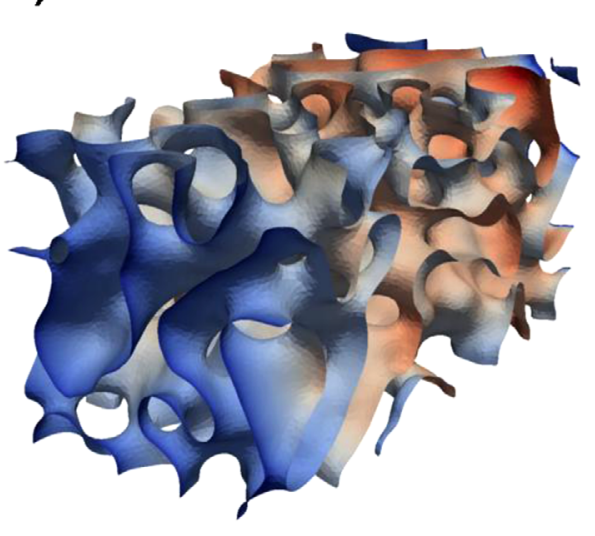

Elec. Dens. $\left(1 / \mathrm{cm}^{3}\right)$

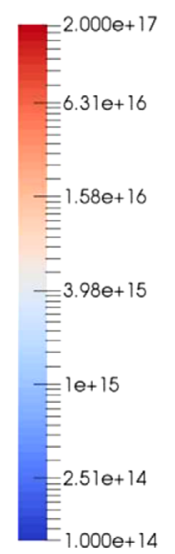

Fig. 2. (a) Structure of the blend, and (b) internal interface showing the electron density.

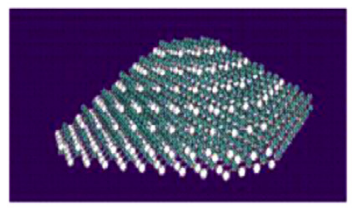

(a)

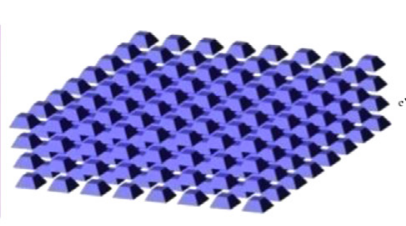

(b)

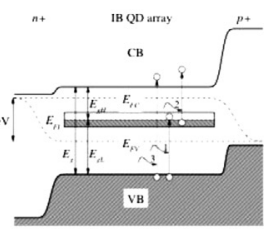

(c)

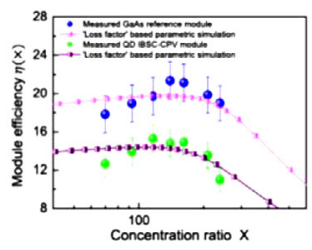

(d)

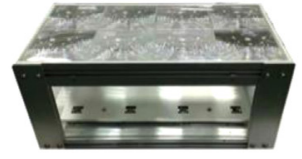

(e)

Fig. 3. Multi-scale approach to the modeling of QD based intermediate band solar cells (IBSC): (a) constituent materials of individual QD is treated at the atomistic quantum mechanical level [59,62,66], (b) array of QD as an absorbing material of the IBSC is treated at semi-empirical mesoscopic quantum mechanical level [20,72], (c) using methods of quantum engineering from (b) the material with new targeted functionality is designed [119], (d) such QD array material is described and the device level by drift diffusion model informed from stages (b and c) in order to predict the IV characteristics and efficiencies of IBSC [20,73], (e) the design is ultimately leading to the fabrication of actual CPV IBSC module consisting of 4 IBSC [22,122].

Figure 2a shows as an example a $100 \mathrm{~nm}$ thick blend obtained via the described procedure, while in Figure $2 \mathrm{~b}$ the corresponding internal interface is presented showing the electron density at the interface [116].

\subsection{Intermediate band solar cells}

In order to increase the efficiency of solar cells, the principal aim must be to make better use of the solar spectrum $[18,117,118]$. One such improvement is to take advantage of the incident photons with sub-band gap energy to be absorbed and contribute to increase photo-current, while in the same time the output voltage of the device would ideally be preserved at its maximal value that is determined by the largest $\mathrm{E}_{\mathrm{g}}$ that exists in the system (i.e., host material energy gap). A possible solution to that problem emerged in the form of the intermediate band solar cell (IBSC) scheme $[119,120]$. The limiting efficiency of the IBSC concept for full concentration and at room temperature is $63.2 \%$, with optimized absorption energies at $\sim 1.2 \mathrm{eV}, \sim 0.7 \mathrm{eV}$ and $\sim 1.9 \mathrm{eV}[119]$, therefore significantly overcoming the Shockley-Queisser limit of $40.7 \%$ for a conventional single-gap SC under the same operating conditions.

Conceptually, an IBSC is manufactured by sandwiching an intermediate band (IB) material between two selective contacts, of $p$ and of $n$ type (see Fig. 3). The IB material is characterized by the existence of an electronic energy band of allowed states within the conventional energy band gap Eg of the host material, splitting it into two sub-gaps, $\mathrm{E}_{\mathrm{gL}}$ and $\mathrm{E}_{\mathrm{gH}}$. This band allows the creation of additional electron-hole pairs from the absorption of two sub-band gap energy photons. Under this assumption, first photon (1) pumps an electron from the valence band (VB) to the IB, and a second photon (2) pumps an electron from the IB to the conduction band (CB). To this end, it is necessary that the IB is half-filled with electrons so that it can supply electrons to the $\mathrm{CB}$ as well as receive them from the VB. This two-photon absorption process is illustrated in Figure 2 and has been experimentally detected in IBSCs based on quantum dots [121]. The electron-hole pairs generated in this way add up to the 
conventionally generated ones by the absorption of a single photon (3), the third one, pumping an electron from the VB to the CB. Therefore, the photocurrent of the solar cell, and ultimately its efficiency, are enhanced since this increment in photocurrent occurs without degradation of the output voltage of the cell. The output voltage is given by the split between electron and hole quasi-Fermi levels, $\mathrm{E}_{\mathrm{FC}}$ and $\mathrm{E}_{\mathrm{FV}}$, that is still limited by the total band gap $\mathrm{E}_{\mathrm{g}}$. The robustness of the IBSC concept allows finding various energy gap combinations that provide for very similar efficiency. This is of particular importance for QD based designs as it opens up a much larger design space for IB solar cells.

\subsection{Multijunction solar cells}

Thermalization loss in solar cells as the main limit on efficiency has one classic solution, which is the multijunction solar cell (MJSC) [123]. Put succinctly, this design absorbs photons in semiconductor regions with bandgaps equal to the photons' energies, thereby eliminating thermalization. The theoretical limiting efficiency in this case is $86.6 \%$ [124]. Two main approaches are mechanical stacking where sub-cells are opticaly coupled, and monolithic integration where subcells are coupled in series optically and electrically.

The fundamental multiscale questions linking the research topics in this field is the mechanical, electronic, and optical coupling between the subcells. On the structural coupling front is the integration of heterogeneous materials, including for example III-V semiconductor integration on $\mathrm{Si}$ where atomistic to device scales are linked by material property modifications due to heterojunctions including strain effects, and to nanostructuring including quantum scale effects. This section sketches the technological issues before summarizing activities in MultiscaleSolar addressing these optical and electronic coupling issues.

In monolithic configuration, subcells are connected in series rather than in parallel. As a consequence, all subcell photocurrents must be equal at the operating point for maximum efficiency, requiring careful current-matching. A second consequence is that compatible materials must be found, that is, semiconductor materials of successively smaller bandgaps which can be grown in stacks. The major incompatibility is in many cases a substantial difference in semiconductor lattice constants, which leads to unacceptably high densities of defects in most materials. While a detailed discussion of this is beyond the scope of this article, we only mention that lattice matched materials combinations dominate this field, while lattice-mismatched solutions using sacrificial relaxed buffer layers achieve results that are nearly equal to the best lattice matched results. We mention finally the use of nanostructured materials in the guise of superlattices, where strain balancing between lattice-mismatched layers may also be used as a solution. An example of this is the multiple-quantum-well MJSC. At present, the series connected monolithic MJSC design is by far the most efficient design available.

Within the consortium, MJSCs are a priority for a number of partners. While a detailed review of these activities is beyond the scope of this article, we mention activities in this field, which are underway. The first is a three terminal device combining high efficiency silicon cells and nanowire layers. The physical novelty of the proposed structure being developed is derived from concepts of front surface passivation combined with band-gap engineering and light scattering and is in the early stages of development.

A second silicon based approach is the development of III-V/Si tandem and triple junction structures [125]. This uses innovative defect-free III-V on $\mathrm{Si}$ growth methods [126]. Current activity within MultiscaleSolar is exploring participation by MultiscaleSolar partners in order to develop optical and transport questions due to the nanostructured nature of the III-V component in the proposed structure. A third active approach is the monolithic ultra-high-efficiency approach being developed by partners working on triple junction [127] and four junction solar cells.

Recently, we have also developed an automated tool for the design of MJSCs, in particular for III-V materials technology, and for efficiency optimization, following the paradigm of heuristic global optimization methods based on genetic algorithms [128].

We conclude this brief summary by noting that, guided by theoretical studies (see for example [129]), the novel materials studied from multiscale perspectives are being studied for multijunction designs. This includes both organic materials and the rapidly developing field of perovskite solar cells, in combination in particular with Si. This field of multijunction solar cells, being the designs achieving efficiencies over $45 \%$ (albeit under concentration) are a major growth area from the perspective of materials as well as next generation nanostructured designs.

\subsection{Multi Exciton Generation solar cells}

In a standard solar cell, all of the energy of an absorbed photon in excess of the effective bandgap of the material is dissipated as heat and essentially wasted. In colloidal quantum dots (QD) (for example, made of CdSe, CdTe, PbSe etc.), this excess photon energy can be utilized via a process known as Multi Exciton Generation (MEG) or direct carrier multiplication (CM). In this process, the high-energy photon creates a high-energy exciton that can decay into a biexciton. For this process to occur, and under the assumption that the electron mass is much lower than the hole mass, the energy of the exciton has to be at least twice as big as the energy of the effective optical gap, i.e., $E_{\mathrm{en}}-E_{\mathrm{h} 0} \geq 2\left|E_{\mathrm{e}}-E_{\mathrm{h} 0}\right|$, where $e 0$ and $h 0$ denote the electron and hole ground states and $e n$ is a state higher in the conduction band. This allows for greater utilization of highenergy photons and dramatically increases solar cell efficiency. The MEG process competes with other radiative and nonradiative recombination and relaxation processes, most of all with Auger cooling [130-132].

Within a simplified model, optical excitation of a QD preserves the symmetry of the wave function and, hence, both a photoexcited electron and a hole are characterized by the same set of quantum numbers that determine the 
angular momentum $(l)$ and the number of nodes in its radial component $(n)$ [133]. As a result, the energies of photoexcited electrons $\left(E_{\mathrm{e}}\right)$ and holes $\left(E_{\mathrm{h}}\right)$ indicates that the energy of a photon in excess of the energy gap, $\left(h_{w}-E_{g}\right)$, is distributed between the electron and the hole in inverse proportion to their effective masses $\mathrm{m}_{\mathrm{e}}$ and $\mathrm{m}_{\mathrm{h}}$, i.e. $E_{\mathrm{e}} /$ $E_{\mathrm{h}}=m_{\mathrm{h}} / m_{\mathrm{e}}$.

Energy conservation requires, that the promotion of the secondary electron across the energy gap can only occur if the greater of two energies $E_{\mathrm{e}}$ and $E_{\mathrm{h}}$ is equal to the gap $E_{\mathrm{g}}$, which leads to the following expression for the CM threshold [134]:

$$
E_{\mathrm{CM}}=E_{\mathrm{g}}\left(2+\frac{m_{\mathrm{e}}}{m_{\mathrm{h}}}\right) .
$$

In the specific case of $m_{\mathrm{e}}=m_{\mathrm{h}}$, it predicts a CM at a threshold of $3 E_{\mathrm{g}}$. This value is smaller than for bulk semiconductors $\left(4 E_{\mathrm{g}}\right)$, which is a direct consequence of the fact that for QDs the secondary-electron excitation step is not subject to translational momentum conservation.

To further increase the solar cell efficiency, it is necessary to optimize the shape and composition of the QDs in order to maximize the ratio of MEG to cooling processes. Theoretical predictions indicate that MEG has the potential to enhance the efficiency of a single-gap cell from $33 \%$ to $44 \%[135,136]$. Full realization of this potential requires that the energy threshold for MEG be minimized. An attractive interaction between excitons reduces the threshold by the biexciton binding energy $\mathrm{B}_{\mathrm{Xx}}$, but this has been found to be small $(-10 \mathrm{meV})$ for type-I QDs.

Colloidal type-II CdSe/CdTe QDs, offer extra degree of freedom in designing MEG devices [137,138]. Previous calculations of BXX in type-II CdSe/CdTe QDs, have found a large repulsion between excitons, while experiment suggests the opposite, i.e., stronger attraction between excitons in the biexciton. To resolve this ambiguity and to gain deeper insight into the excitonic structure of colloidal core/shell CdSe/CdTe type-II QDs, many-body effects like correlation and exchange on the excitonic structure in this class of QDs are investigated in MultiscaleSolar. In addition, the effect of the reduction of the MEG threshold by strong biexciton binding on the ultimate efficiency of an ideal solar cell is object of study. Finally, the extraction of the photogenerated excitons, which has not been well studied so far, is ideally suited for the application of multiscale modeling approaches.

\subsection{Optical modeling at the nanoscale}

Within optical modelling and optimization of solar devices with nanostructured front surfaces, we concentrate on improving existing computational schemes beyond common nanophotonics, including non-classical interaction effects on the ultimate nanometer scale and strong local coupling into large-scale simulations and interaction with, e.g., lattice and hybrid photon-plasmon modes.

Nanostructures and nanoparticle arrays allow efficient forward scattering of incident light, see Figure 4, increasing the exposure of an underlying solar cell to photons. Current research efforts concentrate on functionalized layers in

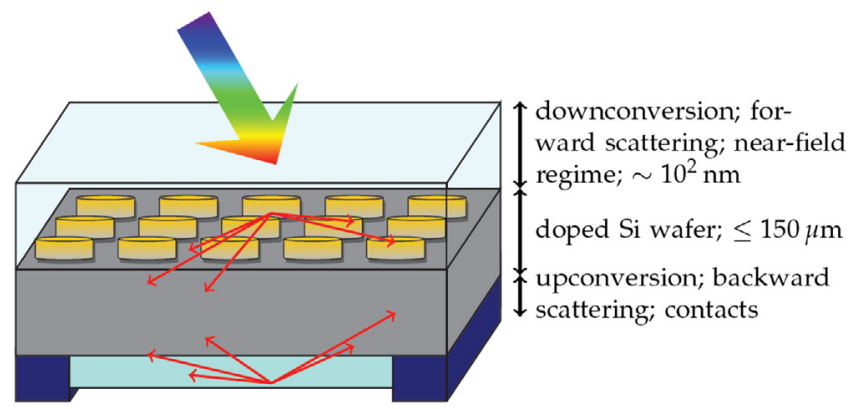

Fig. 4. Next generation solar cells with optically active layers of nanostructures and spectral converters.

addition to standard antireflection coatings to optimize light trapping and exploit local field enhancement of metal nanoparticles via plasmon modes. Plasmon-assisted processes such as direct increase of the charge carrier generation or indirect enhancement of energy conversion effects from neighboring nanocrystal structures have received much interest [139-145].

Fabrication techniques have made tremendous advances in the past decades, in chemical synthesis (etching) [146-150], lithography [151-153], self-assembly through (laser) annealing [152,154,155], and nanoimprint [156159]. Typically, self-assembly and chemical synthesis yield random nanoparticle (NP) layers at reduced costs, while lithography techniques allow for high precision in size, shape, and placement with nanometer resolution [152]. Nanoimprint in particular is a promising route to combine the best of both worlds [158] as it allows keeping costs low using a single imprint template that in turn can be the result of a complex optimization procedure.

Aging and oxidization effects in metals and the shortrange of field effects pose practical challenges. Bio-markers and molecular rulers [160] allow to bring photo-active materials such as rare-earths [143], quantum dots [140,142] and dyes close to NP surfaces. Plasmonic field enhancement as well as scattering effects of nanoparticles can be tuned to enhance photon upconversion in rare-earth ions [161], enabling the conversion of two or more low-energy photons into one higher-energy photon, capable of electron-hole-pair generation in the photovoltaic cell [162]. While upconverting materials are generally placed on the back, downshifting or down converting materials can be placed on the front side to lower the energy of high energy (UV) photons and thereby reducing e.g. thermalization losses [163].

Within computational nanophotonics, a wealth of analytic and numerical tools are available to describe the optical properties of NPs, including arbitrary shapes [148,149,164], particle clusters [165], two-dimensional particle arrays $[164,166,167]$ as well as three-dimensional photonic crystal structures [168].

The key task is the integration of electro-optical effects at the nanoscale and combined coupling of excited nanocrystals with complex energy transfer mechanisms. Mesoscale electron dynamics, surface and thermal effects are not captured in classical electrodynamics and semiclassical approaches are pursued that allow maintaining the 
advantages of computational nanophotonics through extended theories $[167,168,170,171]$. Though such effects are highly localized, optical coupling can lead to an impact on a larger device via retardation and lattice effects [166].

First principle theories can address charge carriers and their mutual interaction with light in detail $[172,173]$. However, the computational effort increases rapidly with the system size. Microscopic theories such as the RPA (Random Phase Approximation) allow investigating fundamental damping mechanisms arising from electron scattering in the bulk material, with the particle surface and with other electrons. Moreover, it allows addressing electron irradiation effects stemming from the accelerated movement of the oscillating electrons forming the plasmon excitation [170,171,174,175].

Spatial dispersion of electron-electron coupling has been studied in semiclassical methods mostly with the hydrodynamic approach $[169,176,177]$, where the dynamics of the electron plasma is separated from polarization effects of bound electrons. This theory yields an additional wave solution, longitudinal in character, and can be solved for different geometries leading to nonlocal extensions of e.g. Mie and Fresnel coefficients [169,178]. The main observations of nonlocal theories in nanosized particles are a blueshift of the plasmon resonance with respect to the common local approximation and plasmon broadening.

The advantage in semiclassical models is their mostly analytic formulation and thus compatibility with existing numerical procedures.

Multiple scattering techniques [165] allow studying particle clusters based on the scattering matrix of a specified particle type [179]. This is in particular interesting for layers with random distributions [152]. The scattering matrix for NPs of arbitrary shapes can be obtained via e.g. BEM (Boundary Element Method), DDA (Discrete Dipole Approximation) or FEM (Finite Element Method) $[143,148,164]$. Extensions for devices and large-scale nanostructures including the aforementioned mesoscale electron dynamics were studied [180].

Several theoretical approaches exist to describe nanostructured layers [168]. Complex layered systems are best modelled within a scattering or transfer matrix approach that for homogeneous layers relies on Fresnel equations. These are available with quantum corrections [178]. For dielectric particles, the RCWA (Rigorous Coupled Wave Analysis) or FMM (Fourier Modal Method) is a fast and reliable computational approach $[167,181]$ that casts the electromagnetic wave equation into an eigenvalue problem via expansion in plane waves and Fourier transform of material parameters. Here again, formulations including quantum corrections exist [166]. These can be coupled with DD equations [91,107] as discussed in previous sections.

Metal NPs, however, pose limitations to these methods due to the high refractive index contrast with their environment. FDTD (Finite Domain Time Difference) and FEM are fully numerical alternative tools to investigate complex architectures. Hence, we investigate alternative materials with plasmonic properties such as conductive nitrides [182].

\section{Conclusions}

In this article we have collected together the four complementary, relevant scales from the atomistic up to industrial scale, sketched the progress and remaining challenges on these length scales as well as interactions between research in these areas yielding a multiscale research analysis of next generation photovoltaic energy concepts. We have sketched the main issues and solutions obtained, illustrated by selected examples of results obtained.

Concerning the atomic scale, we have seen that the central issues are two fold, involving both issues of the specific level of theory (DFT, corrections thereto, time dependent generalizations for optical properties and so on), and computational constraints. These methodological issues can in part be solved by coupling to mesoscopic models. The work reported has led to new Brillouin zone interpolation schemes significantly reducing computational requirements, while extending calculations to physical properties. The properties reported include calculations of optical, transport, and electromechanical constants from atomic scale materials modelling which have been compared to microstructural characterization. Solutions to the main issue of materials properties modelling and parameterization as output to other scales has been demonstrated as a result.

The main issue on the mesoscopic and nanostructure scale is the propagation of materials properties based on inputs from atomic scale modeling up to the device scale. The work reported here successfully developed tailored solutions for important structures defined at the device scale, but also by industrial level requirements. These tailored solutions consist of parameterizing material properties from ab-initio atomic models and applying a number of techniques (principally KMC and NEGF) to compute optical and transport rates for the structures defined on device level. The issues for the mesosopic scale of acting as a means of implementing atomic level outputs for application at device level have, in the work reported, shown progress in multiscale modelling at device level as a result, reported in detail in resulting publications referred to in the main text.

We have seen that the main focus of research at the device scale is on 3rd generation photovoltaics and, naturally, nanostructure states. Issues in modelling include both analytical and numerical TCAD approaches, where the perspective of the device level is helping to define structures of interest for the mesoscopic or nanostructure scale modelling. As a consequence, device modelling reported in this paper has benefited from coupling nanostructure state models (including KMC and NEGF) with classical DD models. This still preliminary coupling is the subject of ongoing work and future development.

From the point of view of industrial perspectives, the over-riding issue is the identification of promising new routes bearing potential of positive societal impacts. Identification of promising and viable technologies is at the root of the modelling hierarchy, ranging from device level down to the atomistic material modelling. Therefore, we have presented methods put into practice to identify 
relevant industrial trends. As reported in the main text, this has included elements of industrial activity within MultiscaleSolar on modelling and fabrication fronts, but has most importantly taken the form of surveys of industrial trends. For this purpose, on-site studies by researchers from industrial and academic contexts has proven as valid tool, informing the choice of structures investigated on the device scale in particular.

In this review of the main issues and the main achievements of MultiscaleSolar, we see a developing knowledge exchange between researchers working on different scales, and in particular between mesoscale and macroscale device physics. On the materials based topics, we see input from ab-initio materials properties modeling feeding in to both optical and transport simulation for next generation structures, with the microscale research providing direction on the development of materials and characterization feeding through the mesoscale and macroscale up to the application-oriented research on industrial perspectives.

In addition to the scientific and technical work carried out, MultiscaleSolar, through the interactions shown by the contributors to this paper, is combining research expertise and infrastructure across the European Union. The complementarity of groups in multiscale analysis is thrown into sharp focus by the range of expertise which is to be found in different academic and industrial traditions.

However, although this joint paper shows progress on the range of organic and inorganic materials, and on tried and tested solar cell designs as well as third generation concepts, we conclude that there remains much to be done. The theoretical efficiency limits for third-generation concepts remain far above what has been achieved to date. One of the expected outcomes of MultiscaleSolar is bringing multiscale techniques to bear to understanding what the roadblocks to achieving these efficiencies are. Ultimately to either injecting greater realism in achievable efficiencies, or identifying routes to achieving them in these emerging technologies.

The authors are grateful for the financial support by the COST Action MP1406 "MultiscaleSolar."

\section{Author contribution statement}

Urs Aeberhard, Matthias Auf der Maur, Neil Beattie, James Connolly, Christin David, Alessio Gagliardi, JeanPaul Kleider, Jean-Louis Lazzari, Laurent Pedesseau, Stanko Tomić and Javad Zarbakhsh contributed substantially to the writing. Matthias Auf der Maur coordinated the manuscript preparation and submission. All authors contributed to the discussions, ongoing work and presented results.

\section{References}

1. https://ec.europa.eu/energy/en/topics/renewable-energy/ renewable-energy-directive

2. European Commission, The roadmap for transforming the EU into a competitive, low-carbon economy by 2050, https://ec.europa.eu/clima/citizens/eu_en
3. http://eur-lex.europa.eu/legal-content/EN/TXT/ HTML/?uri=CELEX:52016PC0767R(01)\&from =EN

4. B. Obama, Science 355, 126 (2017)

5. http://www.iea-pvps.org

6. http://www.ren21.net

7. https://ec.europa.eu/jrc/en

8. A. Gambhir et al., Solar Energy Mater. Solar Cells 156, 49 (2016)

9. www.iea.org, Renewables 2017, Analysis \& Forecasts to 2022, Executive Summary

10. J. Zhao, A. Wang, M.A. Green, Prog. Photovolt. 7, 471 (1999)

11. D.D. Smith, G. Reich, M. Baldrias, M. Reich, N. Boitnott, G. Bunea, in 2016 IEEE 43rd Photovoltaic Specialists Conference (PVSC) (2016), p. 3351

12. S.W. Glunz, F. Feldmann, A. Richter, M. Bivour, C. Reichel, H. Steinkemper, J. Benick, M. Hermle, in Proc. of the 31st European Photovoltaic Solar Energy Conference and Exhibition (2015), p. 259

13. D. Adachi, J.L. Hernandez, K. Yamamoto, Appl. Phys. Lett. 107, $233506(2015)$

14. M. Taguchi, A. Yano, S. Tohoda, K. Matsuyama, Y. Nakamura, T. Nishiwaki, K. Fujita, E. Maruyama, IEEE J. Photovolt. 4, 96 (2014)

15. K. Yoshikawa, H. Kawasaki, W. Yoshida, T. Irie, K. Konishi, K. Nakano, T. Uto, D. Adachi, M. Kanematsu, H. Uzu, K. Yamamoto, Nat. Energy 2, 17032 (2017)

16. https://www.nrel.gov/pv/assets/images/efficiency-chart.png

17. W. Shockley, H.J. Queisser, J. Appl. Phys. 32, 510 (1961)

18. R.R. King, Nat. Photon. 2, 284 (2008)

19. J. Even et al., Int. J. Photoenergy 2014, 649408 (2014)

20. S. Tomić, T. Sogabe, Y. Okada, Prog Photovolt: Res. Appl. 23, 546 (2015)

21. U. Aeberhard, IEEE J. Photovolt. 6, 654 (2016)

22. T. Sogabe, Y. Shoji, M. Ohba, K. Yoshida, R. Tamaki, H.-F. Hong, C.-H. Wu, C.-T. Kuo, S. Tomić, Y. Okada, Sci Rep. 4, $4792(2014)$

23. M. Auf der Maur, J. Green Eng. 5, 133 (2016)

24. U. Aeberhard et al., J. Green Eng. 5, 11 (2016)

25. J.P. Connolly et al., Renew. Energy Environ. Sustain. 1, 6 (2016)

26. http://multiscalesolar.eu/

27. I. Lucci, S. Charbonnier, L. Pedesseau, M. Vallet, L. Cerutti, J.-B. Rodriguez, E. Tournié, R. Bernard, A. Létoublon, N. Bertru, A. Le Corre, S. Rennesson, F. Semond, G. Patriarche, L. Largeau, P. Turban, A. Ponchet, C. Cornet, Phys. Rev. Mater. 2, 060401 (2018)

28. W. Kohn, L.J. Sham, Phys. Rev. 140, A1133 (1965)

29. A.D. Becke, J. Chem. Phys. 98, 5648 (1993)

30. S. Tomić, B. Montanari, N.M. Harrison, Physica E 40, 2125 (2008)

31. J. Heyd, G. Scuseria, M. Ernzerhof, J. Chem. Phys. 118, 8207 (2003)

32. L. Hedin, Phys. Rev. 139 A796 (1965)

33. L. Bernasconi, S. Tomić, M. Ferrero, M. Rérat, R. Orlando, R. Dovesi, N.M. Harrison, Phys. Rev. B 83, 195325 (2011)

34. H. Bethe, E. Salpeter, Phys. Rev. 84, 1232 (1951)

35. C. Rödl, T. Sander, F. Bechstedt, J. Vidal, P. Olsson, S. Laribi, J.F. Guillemoles, Phys. Rev. B 92, 045207 (2015)

36. S.X. Tao, X. Cao, P.A. Bobbert, Sci. Rep 7, 14386 (2017)

37. G. Kresse, J. Furthmüller, Comput. Mat. Sci. 6, 15 (1996)

38. G. Kresse, J. Furthmüller, Phys. Rev B 54, 11169 (1996)

39. X. Gonze, J.-M. Beuken, R. Caracas, F. Detraux, M. Fuchs, G.-M. Rignanese, L. Sindic, M. Verstraete, G. Zerah, F. 
Jollet, M. Torrent, A. Roy, M. Mikami, Ph. Ghosez, J.-Y. Raty, D.C. Allan, Comput. Mater. Sci. 25, 478 (2002)

40. J. Soler, E. Artacho, J. Gale, A. García, J. Junquera, P. Ordejón, D. Sánchez-Portal, J. Phys: Condens. Matter 14, 2745 (2002)

41. P. Giannozzi, S. Baroni, N. Bonini, M. Calandra, R. Car, C. Cavazzoni, D. Ceresoli, G.L. Chiarotti, M. Cococcioni, I. Dabo, A. Dal Corso, S. Fabris, G. Fratesi, S. de Gironcoli, R. Gebauer, U. Gerstmann, C. Gougoussis, A. Kokalj, M. Lazzeri, L. Martin-Samos, N. Marzari, F. Mauri, R. Mazzarello, S. Paolini, A. Pasquarello, L. Paulatto, C. Sbraccia, S. Scandolo, G. Sclauzero, A.P. Seitsonen, A. Smogunov, P. Umari, R.M. Wentzcovitch, J. Phys: Condens. Matter 21, 395502 (2009)

42. I.J. Bush, S. Tomić, B. G. Searle, G. Mallia, C.L. Bailey, B. Montanari, L. Bernasconi, J.M. Carr, N. M. Harrison, Proc. R. Soc. Lond. A: Math. Phys. Eng. Sci. 467, 2112 (2011)

43. S.J. Clark, M.D. Segall, C.J. Pickard, P.J. Hasnip, M.I.J. Probert, K. Refson, M.C. Payne, Z. Kristallogr. Cryst. Mater. 220, 567 (2005)

44. J. Even, L. Pedesseau, M.A. Dupertuis, J.M. Jancu, C. Katan, Phys. Rev. B 86, 205301 (2012)

45. J. Even, L. Pedesseau, J.M. Jancu, C. Katan, J. Phys. Chem. Lett. 4, 2999 (2013)

46. J. Even, L. Pedesseau, C. Katan, J. Phys. Chem. C 118, 11566 (2014)

47. L. Pedesseau, D. Sapori, B. Traore, R. Robles, H-H Fang, M. Loi, H. Tsai, W. Nie, J.-C. Blancon, A. Neukirch, S. Tretiak, A. D. Mohite, C. Katan, J. Even, M. Kepenekian, ACS Nano 10, 9776 (2016)

48. P. Czaja, M. Celino, S. Giusepponi, M. Gusso, U. Aeberhard, in Lecture Notes in Computer Science (Springer, Cham, 2017), Vol. 10164, pp. 111-124

49. C. Persson, Y.-J. Zhao, S. Lany, A. Zunger, Phys. Rev. B 72 , $035211(2005)$

50. C. Persson, A. Zunger, Phys. Rev. Lett. 91, 266401 (2003)

51. C.L. Bailey, L. Liborio, G. Mallia, S. Tomić, N.M. Harrison, Phys. Rev. B 81, 205214 (2010)

52. L.M. Liborio, C. L. Bailey, G. Mallia, S. Tomic, N.M. Harrison, J. Appl. Phys. 109, 023519 (2011)

53. S. Tomić, L. Bernasconi, B.G. Searle, N.M. Harrison, J. Phys. Chem. C 118, 14478 (2014)

54. J. Vidal, S. Botti, J.-F. Guillemoles, L. Reining, Phys. Rev. Lett. 104, 056401 (2010)

55. M. Lundie, Z. Šljivančanin, S. Tomić, J. Mater. Chem. C 3, $7632(2015)$

56. E.P. O'Reilly, A. Lindsay, S. Tomić, M. Kamal-Saadi, Semicond. Sci. Technol. 17, 870 (2002)

57. J. Even, F. Doré, C. Cornet, L. Pedesseau, A. Schliwa, D. Bimberg, Appl. Phys. Lett. 91, 122112 (2007)

58. S. Boyer-Richard, F. Raouafi, A Bondi, L. Pedesseau, C. Katan, J.-M. Jancu, J. Even, Appl. Phys. Lett. 98, 251913 (2011)

59. C. Van de Walle, R. Martin, Phys. Rev. B 35, 8154 (1987)

60. J. Pal, G. Tse, V. Haxha, M.A. Migliorato, S. Tomić, Phys. Rev. B 84, 085211 (2011)

61. S. Tomić, N. Vukmirović, J. Appl. Phys. 110, 053710 (2011)

62. M. Auf Der Maur, M. Povolotskyi, F. Sacconi, A. Pecchia, G. Romano, G. Penazzi, A. Di Carlo, TiberCAD: Towards multiscale simulation of optoelectronic devices, 2008 International Conference on Numerical Simulation of Optoelectronic Devices (NUSOD), Nottingham (2008), pp. $43-44$
63. K. Berland, C. Persson, Comput. Mater. Sci. 134, 17 (2017)

64. F.C.M. Spoor, S. Tomic, A.J. Houtepen, L.D.A. Siebbeles, ACS Nano 11, 6286 (2017)

65. V. Fiorentini, F. Bernardini, F. Della Sala, A. Di Carlo, P. Lugli, Phys. Rev. B 60, 8849 (1999)

66. J.M. Jancu, R. Scholz, F. Beltram, F. Bassani, Phys. Rev. B 57, 6493 (1998)

67. S. Boyer-Richard, C. Katan, B. Traoré, R. Scholz, J.M. Jancu, J. Even, J. Phys. Chem. Lett. 7, 3833 (2016)

68. A. Marronnier, G. Roma, S. Boyer-Richard, L. Pedesseau, J.-M. Jancu, Y. Bonnassieux, C. Katan, C. Stoumpos, M. Kanatzidis, J. Even, ACS Nano 12, 3477 (2018)

69. U. Aeberhard, J. Comput. Electron. 10, 394 (2011)

70. P.K. Watkins, A.B. Walker, G.L.B. Verschoor, Nano Letters 5, 1814 (2005)

71. S. Tomić, A.G. Sunderland, I.J. Bush, J. Mater. Chem. 16, 1963 (2006)

72. N. Vukmirović, Z. Ikonić, D. Indjin, P. Harrison, Phys. Rev. B 76, 245313 (2007)

73. A. Berbezier, U. Aeberhard, Phys. Rev. Appl. 4, 044008 (2015)

74. P. Peumans, S. Uchida, S.R. Forrest, Nature 425, 158 (2003)

75. P.K. Watkins, A.B. Walker, G.L.B. Verschoor, Nano Lett. 5, $1814(2005)$

76. P. Kordt, J.JM van der Holst, M. Al Helwi, W. Kowalsky, F. May, A. Badinski, C. Lennartz, D. Andrienko, Adv. Funct. Mater. 25, 1955 (2015)

77. M. Mladenović, N. Vukmirović, J. Phys. Chem. C 119, 41 (2015)

78. M. Mladenović, N. Vukmirović, J. Phys. Chem. C 120, 18895 (2016)

79. V. Janković, N. Vukmirović, Phys. Rev. B 92, 235208 (2015)

80. V. Janković, N. Vukmirović, Phys. Rev. B 95, 075308 (2017)

81. V. Donchev, M. Milanova, J. Lemieux, N. Shtinkov, I.G. Ivanov, J. Phys: Conf. Ser. 700, 012028 (2016)

82. V. Donchev, I. Asenova, M. Milanova, D. Alonso-Álvarez, K. Kirilov, N. Shtinkov, I.G. Ivanov, S. Georgiev, E. Valcheva, N. Ekins-Daukes, J. Phys: Conf. Ser. 794, 012013 (2017)

83. M. Milanova, V. Donchev, K. Kostov, D. Alonso-Álvarez, E . Valcheva, K. Kirilov, I. Asenova, I.G. Ivanov, S. Georgiev, N. Ekins-Daukes, Semicond. Sci. Technol. 32, 085005 (2017)

84. http://www.silvaco.com

85. http://www.nextnano.com

86. http://www.tiberlab.com

87. E. Garnett, P. Yang, Nano Lett. 10, 1082 (2010)

88. S. Masudy-Panah, K. Radhakrishnan, H.R. Tan, R. Yi, T.I. Wong, G.K. Dalapati, Solar Energy Mater. Solar Cells 140, $266(2015)$

89. S. Heo, G. Seo, Y. Lee, D. Lee, M. Seol, J. Lee, J.-B. Park, K. Kim, D. Yun, Y.S. Kim, J.K. Shin, T.K. Ahn, M.K. Nazeeruddin, Energy Environ. Sci. 10, 1128 (2017)

90. B. Weiler, A. Gagliardi, P. Lugli, J. Phys. Chem. C 120, 10062 (2016)

91. A. Gagliardi et al., Nanoscale 7, 1136 (2015)

92. M. Auf der Maur, A. Pecchia, G. Penazzi, W. Rodrigues, A. Di Carlo, Phys. Rev. Lett. 116, 027401 (2016)

93. S. Tomić, J. Pal, M.A. Migliorato, R.J. Young, N. Vukmirović, ACS Photonics 2, 958 (2015)

94. https://www.edf.fr

95. https://www.chimie-paristech.fr/fr/la_recherche/IRDEP 
96. https://www.toyota-europe.com

97. http://www.horiba.com/uk/

98. http://www.solarcapturetechnologies.com

99. http://www.3gsolar.com

100. http://www.bisol.si

101. http://www.semilab.com

102. http://www.quantumbase.co.uk

103. http://www.nanotechnologysolar.com

104. S.G. Hashmi, M. Özkan, J. Halme, S.M. Zakeeruddin, J. Paltakari, M. Grätzel, P.D. Lund, Energy Environ. Sci. 9, 2453 (2016)

105. E. Da Como, F. De Angelis, H. Snaith, A. Walker, Unconventional thin film photovoltaics, RCS Energy and Environmental Series (Royal Society of Chemistry, 2016)

106. A. Gagliardi, A. Abate, ACS Energy Lett. 3, 163 (2017)

107. A. Gagliardi, M. Auf der Maur, D. Gentilini, A. Di Carlo, J. Comput. Electron. 10, 424 (2011)

108. A.H. Fallahpour, et al., J. Comput. Electron. 13, 933 (2014)

109. A. Gagliardi, M. Auf der Maur, A. Di Carlo, IEEE J. Quantum Electron. 47, 1214 (2011)

110. T. Albes, P. Lugli, A. Gagliardi, IEEE Trans. Nanotechnol. 15, $281(2016)$

111. https://www.sne.ei.tum.de/en/research/

112. P. Peumans, S. Uchida, S.R. Forrest, Nature 425, 158 (2003)

113. G. Taubin, Proc. ACM SIGGRAPH 95, 351 (1995)

114. M. Desbrun, M. Meyer, P. Schroeder, A.H. Barr, Proc. ACM SIGGRAPH 99, 317 (1999)

115. C. Geuzaine, J.-F. Remacle, Int. J. Numer. Methods Eng. 79, 1309 (2009)

116. A. Gagliardi, S. Wang, T. Albes, Org. Electron. 59, 171 (2018)

117. M. Green, Prog. Photovolt. 9, 123 (2001)

118. A. Nozik, Physica E 14, 115 (2002)

119. A. Luque, A. Marti, Phys. Rev. Lett. 78, 5014 (1997)

120. A. Luque, A. Marti, Adv. Mater. 22, 160 (2010)

121. S. Asahi, H. Teranishi, K. Kusaki, T. Kaizu, T. Kita, Nat. Commun. 8, 14962 (2017)

122. N.S. Beattie, P. See, G. Zoppi, P.M. Ushasree, M. Duchamp, I. Farrer, D.A. Ritchie, S. Tomić, ACS Photonics 4, 2745 (2017)

123. E.D. Jackson, Trans. Conf. Use Sol. Energy 5, 122 (1958)

124. A. Martí, Sol. Energy Mater. Sol. Cells 33, 213 (1994)

125. J.P. Connolly et al., Prog. Photovolt. Res. Appl. 22, 810 (2014)

126. C. Renard et al., Sci. Rep. 6, 25328 (2016)

127. A. Tukiainen et al., Prog. Photovolt. Res. Appl. 24, 914

128. S. Čičić, S. Tomić, Sol. Energy Mater. Sol. Cells 181, 30 (2018)

129. R. Lachaume et al., Sol. Energy Mater. Sol. Cells 166, 276 (2017)

130. L.-W. Wang, M. Califano, A. Zunger, A. Franceschetti, Phys. Rev. Lett. 91, 056404 (2003)

131. M. Califano, A. Zunger, A. Franceschetti, Appl. Phys. Lett. 84, 2409 (2004)

132. S. Tomic, J.M. Miloszewski, E.J. Tyrrell, D.J. Binks, IEEE J. Photovol. 6, 179 (2016)

133. A.L. Efros, Sol. Phys. Semicond. 16, 772 (1982)

134. R.D. Schaller, M.A. Petruska, V.I. Klimov, Appl. Phys. Lett. 87, 253102 (2005)

135. V.I. Klimov, Appl. Phys. Lett. 89, 123118 (2006)

136. D.J. Binks, Phys. Chem. Chem. Phys. 13, 12693 (2011)

137. M.A. Leontiadou, E.J. Tyrrell, C.T. Smith, D. EspinobarroVelazquez, R. Page, J. Miloszewski, T. Walsh, D. Binks, S. Tomić, Sol. Energy Mater. Sol. Cells 159, 657 (2017)
138. C.T. Smith, E.J. Tyrrell, M.A. Leontiadou, J. Miloszewski, T. Walsh, M. Cadirci, R. Page, D. Binks, St. Tomić, Sol. Energy Mat. Sol. Cells 158, 160 (2016)

139. E. Klampaftis, B.S. Richards, Prog. Photovolt. Res. Appl. 19, 345 (2010)

140. Z. Yuan, G. Pucker, A. Marconi, F. Sgrignuoli, A. Anopchenko, Y. Jestin, L. Ferrario, P. Bellutti, L. Pavesi, Solar Energy Mater. Solar Cells 95, 1224 (2011)

141. D. Alonso-Âlvarez, D. Ross, E. Klampaftis, K.R. McIntosh, S. Jia, P. Storiz, T. Stolz, B.S. Richards, Prog Photovolt: Res. Appl. 23, 479 (2014).

142. S. Gardelis, A.G. Nassiopoulou, Appl. Phys. Lett. 104, $183902(2014)$

143. S.R. Johannsen, S.P. Madsen, B.R. Jeppesen, J.V. Nygaard, B. Julsgaard, P. Balling, A.N. Larsen, Appl. Phys. Lett. 106, 053101 (2015)

144. D. Fuertes Marrón, E. Barrigón, M. Ochoa, I. Artacho, Phys. Rev. Appl. 6, 014001 (2016)

145. S. Gardelis, V. Gianneta, A. Nassiopoulou, J. Luminescence 170, 282 (2016)

146. P. Lalanne, G.M. Morris, NanoTechnology 8, 53 (1997)

147. P.S. Kumar, I. Pastoriza-Santos, B. Rodríguez-González, F. J.G. de Abajo, L.M. Liz-Marzán, NanoTechnology 19, 015606 (2008)

148. V. Myroshnychenko, J. Rodríguez-Fernández, I. PastorizaSantos, A.M. Funston, C. Novo, P. Mulvaney, L.M. LizMarzán, F.J.G. de Abajo, Chem. Soc. Rev. 37, 1792 (2008)

149. R. Alvarez-Puebla, L.M. Liz-Marzán, F.J.G. de Abajo, J. Phys. Chem. Lett. 1, 2428 (2010)

150. Y. Liu, T. Lai, H. Li, Y. Wang, Z. Mei, H. Liang, Z. Li, F. Zhang, W. Wang, A.Y. Kuznetsov, X. Du, Small 8, 1392 (2012)

151. T. Sakai, N. Nedyalkov, M. Obara, J. Phys. D: Appl. Phys. 40, 2102 (2007)

152. C. David, J.P. Connolly, C. Chaverri Ramos, F.J.G. de Abajo, G. Sánchez Plaza, Sol. Energy Mater. Sol. Cells 109, 294 (2013)

153. K. Wu, T. Rindzevicius, M.S. Schmidt, K.B. Mogensen, S. Xiao, A. Boisen, Opt. Express 23, 12965 (2015)

154. F. Cortés-Juan, C. Chaverri Ramos, J.P. Connolly, C. David, F.J. García de Abajo, J. Hurtado, V.D. Mihailetchi, S. Ponce-Alcántara, G. Sánchez, J. Renew. Sustain. Energy 5, 033116 (2013)

155. N. Kalfagiannis, A. Siozios, D.V. Bellas, D. Toliopoulos, L. Bowen, N. Pliatsikas, W.M. Cranton, C. Kosmidis, D.C. Koutsogeorgis, E. Lidorikis, P. Patsalas, Nanoscale 8, 8236 (2016)

156. O. Watanabe, T. Ikawa, M. Hasegawa, M. Tsuchimori, Y. Kawata, Appl. Phys. Lett. 79, 1366 (2001)

157. A. Sundaramurthy, P.J. Schuck, N.R. Conley, D.P. Fromm, G.S. Kino, W.E. Moerner, Nano Lett. 6, 355 (2006)

158. P. Spinelli, V.E. Ferry, J. van de Groep, M. van Lare, M.A. Verschuuren, R.E.I. Schropp, H.A. Atwater, A. Polman, J. Opt. 14, 024002 (2012)

159. C. David, P. Kühler, F. Javier García de Abajo, J. Siegel, Opt. Express 22, 8226 (2014)

160. M.E. Anderson, M. Mihok, H. Tanaka, L.-P. Tan, M.W. Horn, G.S. McCarty, P.S. Weiss, Adv. Mater. 18, 1020 (2006)

161. H. Lakhotiya, A. Nazir, S.P. Madsen, J. Christiansen, E. Eriksen, J. Vester-Petersen, S.R. Johannsen, B.R. Jeppesen, P. Balling, A. Nylandsted Larsen, B. Julsgaard, Appl. Phys. Lett. 109, 263102 (2016) 
162. J.C. Goldschmidt, S. Fischer, Adv. Opt. Mater. 3, 510 (2015)

163. E. Klampaftis, D. Ross, K.R. McIntosh, B.S. Richards, Sol. Energy Mat. Sol. C 93, 1182 (2009)

164. F.J.G. de Abajo, Rev. Mod. Phys. 79, 1267 (2007)

165. F.J.G. de Abajo, Phys. Rev. B 60, 6086 (1999).

166. C. David, J. Christensen, N.A. Mortensen, Phys. Rev. B 94, 165410 (2016)

167. C. David, Appl. Opt. Appl. Opt. 55, 7980 (2016)

168. J.D. Joannopoulos, S.G. Johnson, J.N. Winn, R.D. Meade, Photonic Crystals: Molding the Flow of Light, 2nd ed. (Princeton University Press, Princeton, New Jersey, USA, 2008)

169. C. Davi, F.J. García de Abajo, J. Phys. Chem. C 115, 19470 (2011)

170. W. Jacak, J. Phys. Chem. C 119, 6749 (2015)

171. W. Jacak, E. Popko, A. Henrykowski, E. Zielony, K. Gwozdz, G. Luka, R. Pietruszka, B. Witkowski, L. Wachnicki, M. Godlewski et al., Solar Energy Mater. Solar Cells 147, 1 (2016)
172. J. Zuloaga, E. Prodan, P. Nordlander, Nano Lett. 9, 887 (2009)

173. R. Esteban, A.G. Borisov, P. Nordlander, J. Aizpurua, Nat. Commun. 3, 825 (2012)

174. J. Jacak, J. Krasnyj, W. Jacak, R. Gonczarek, A. Chepok, L. Jacak, Physi. Rev. B 82, 035418 (2010)

175. K. Kluczyk, W. Jacak, J. Quant. Spectrosc. Radiat. Transf. 168, 78 (2016)

176. C. David, F.J. García de Abajo, ACS Nano 8, 9558 (2014)

177. S. Raza, S.I. Bozhevolnyi, M. Wubs, N.A. Mortensen, J. Phys. Cond. Matter. 27, 183204 (2015)

178. C. David, N.A. Mortensen, J. Christ. Sci. Rep. 3, 2526 (2013)

179. K. Kluczyk, C. David, W.A. Jacak. JOSA B 34, 2115 (2017)

180. C. David, J. Christensen, Appl. Phys. Lett. 10, 261110 (2017)

181. P. Lalanne, G.M. Morris, J. Opt. Soc. Am. A-Opt. Image Sci. Vis. 13, 779 (1996)

182. P. Patsalas et al., Mater. Sci. Eng. R Rep. 123, 1 (2018)

Cite this article as: Tareq Abu Hamed, Nadja Adamovic, Urs Aeberhard, Diego Alonso-Alvarez, Zoe Amin-Akhlaghi, Matthias Auf der Maur, Neil Beattie, Nikola Bednar, Kristian Berland, Stefan Birner, Marco Califano, Ivana Capan, Bostjan Cerne, Irinela Chilibon, James. P. Connolly, Frederic Cortes Juan, Jose Coutinho, Christin David, Knut Deppert, Vesselin Donchev, Marija Drev, Boukje Ehlen, Nicholas Ekins-Daukes, Jacky Even, Laurentiu Fara, David Fuertes Marron, Alessio Gagliardi, Blas Garrido, Violetta Gianneta, Maria Gomes, Jean-Francois Guillemoles, Mircea Guina, Janne Halme, Mateja Hocevar, Lucjan Jacak, Witold Jacak, Zoran Jaksic, Lejo K. Joseph, Spyridon Kassavetis, Vaidotas Kazukauskas, Jean-Paul Kleider, Katarzyna Kluczyk, Radovan Kopecek, Ursa Opara Krasovec, Jean-Louis Lazzari, Efrat Lifshitz, Martin Loncaric, Søren Peder Madsen, Antonio Marti Vega, Denis Mencaraglia, Maria E. Messing, Felipe Murphy Armando, Androula G. Nassiopoulou, Ahmed Neijm, Akos Nemcsics, Victor Neto, Laurent Pedesseau, Clas Persson, Konstantinos Petridis, Lacramioara Popescu, Georg Pucker, Jelena Radovanović, Julio C. Rimada, Mimoza Ristova, Ivana Savic, Hele Savin, Marushka Sendova-Vassileva, Abdurrahman Sengul, José Silva, Ullrich Steiner, Jan Storch, Emmanuel Stratakis, Shuxia Tao, Pavel Tomanek, Stanko Tomić, Antti Tukiainen, Rasit Turan, Jose Maria Ulloa, Shengda Wang, Fatma Yuksel, Jaroslav Zadny, Javad Zarbakhsh, Multiscale in modelling and validation for solar photovoltaics, EPJ Photovoltaics 9, 10 (2018) 